\title{
Short Communication \\ Interleukin-6 gene amplification and shortened survival in glioblastoma patients
}

\author{
A Tchirkov*,1,2,3, T Khalil ${ }^{1,4}$, E Chautard', K Mokhtari ${ }^{5}$, L Véronèse ${ }^{1,2}$, B Irthum ${ }^{4}$, P Vago ${ }^{2}$, J-L Kémény ${ }^{6}$ and \\ P Verrelle ${ }^{1,3}$ \\ 'EA 3846, Université d'Auvergne, Clermont-Ferrand, F-6300I, France; ' Service de Cytogénétique Médicale, UFR Médecine, CHU, Clermont-Ferrand, \\ F-6300 I, France; ${ }^{3}$ Departement de Radiothérapie, Centre Jean Perrin, Clermont-Ferrand, F-630II, France; ${ }^{4}$ Senvices de Neurochirurgie, CHU, Clermont- \\ Ferrand, F-6300 I, France; ${ }^{5}$ Laboratoire de Neuropathologie, Groupe hospitalier Pitie-Salpetriere, Paris, F-750 I3, France; ${ }^{6}$ Laboratoire d'Anatomie \\ Pathologique, CHU, Clermont-Ferrand, F-6300I, France
}

\section{INTRODUCTION}

Interleukin-6 (IL-6) is a pleiotropic cytokine that regulates the immune response, but also plays a role in promoting tumour growth and survival (Trikha et al, 2003; Hodge et al, 2005). In gliomas, the level of $I L-6$ gene expression increases with the grade of malignancy (Rolhion et al, 2001). In glioblastoma multiforme (GBM), the most malignant glioma, amplification/overexpression of the IL-6 gene appears to be a common feature (Tchirkov et al, 2001). Recent genomic array studies have reported that the number of $I L-6$ gene copies was increased in $40-50 \%$ of GBM (Suzuki et al, 2004; Saigusa et al, 2005). It is however unknown whether this alteration could be found in gliomas of lower malignancy grades. The prognostic significance of IL-6 gene amplification in GBM has not yet been determined. To address these issues, we evaluated here $I L-6$ gene amplification using interphase fluorescence in situ hybridisation (FISH) in 53 gliomas representative of various histological types and malignancy grades. In GBM, the results were correlated with patient survival.

\section{MATERIALS AND METHODS}

Glioma samples were taken from the material of surgical resection during the course of standard diagnostic procedure. Histological diagnosis and grading of tumours was consistent with the World Health Organization (WHO) criteria (World Health Organization, 2000). Nine tumours were classified as low-grade gliomas (grade

*Correspondence: Dr A Tchirkov, EA 3846, Université d'Auvergne, Clermont-Ferrand, F-6300 I, France;

E-mail: andrei.tchirkov@cjp.fr

Received 6 October 2006; revised 14 December 2006; accepted 14 December 2006; published online 16 January 2007
I-II), including one pilocytic astrocytoma, three astrocytomas, two oligodendrogliomas and three oligoastrocytomas. Eight tumours were anaplastic gliomas (grade III), including six anaplastic oligoastrocytomas and two anaplastic oligodendrogliomas. The remaining 36 tumours were classified as GBM (grade IV). Patients with malignant gliomas (grade III-IV) were treated by surgery followed by either high-dose carmustine followed by radiotherapy in cases with optimal tumour resection or radiotherapy and concomitant temozolomide followed by monthly cycles of adjuvant temozolomide in cases with partial tumour resection or biopsy alone.

An interphase FISH was performed on frozen sections of gliomas mounted on Fisher Superfrost slides. The $I L-6$ gene probe was designed from the bacterial artificial chromosome clone RP11240H8 (GenBank Accession Number AC073072) kindly provided by Professor Mariano Rocchi (University of Bari, Italy). The IL-6 probe labelled with SpectrumGreen (Vysis/Abbott, Rungis, France) and chromosome 7 cetromeric probe labelled with SpectrumOrange (Vysis/Abbott) were co-hybridised to evaluate simultaneously the number of $I L-6$ gene and chromosome 7 copies. A median of 148 nuclei (116-245, interquartile range) was scored using a Metafer4-MetaCyte microscope scanning system (MetaSystems, Le Cannet, France).

The number of IL-6 mRNA transcripts was assessed using quantitative real-time reverse transcriptase-PCR (qRT-PCR) in the LightCycler system (Roche Diagnostics, Meylan, France) with $I L-6$ specific primers (Tchirkov et al, 2001) and normalised to the expression of a housekeeping gene, $A B L$ (Tchirkov et al, 2003).

Analyses of statistical links between biological and clinical characteristics were performed using standard tests. Overall survival was calculated using the Kaplan-Meier method and survival curves were compared using the log-rank test. A multivariate analysis was performed using the Cox regression model. 


\section{RESULTS}

Using interphase FISH approach, no IL-6 gene amplification was detected in low-grade or anaplastic tumours $(n=17)$, whereas high-level amplification was found in 15 out of 36 (41.7\%) GBM (Figure $1 \mathrm{~A}$ and $\mathrm{B}$ ). The percentage of nuclei with amplification events varied between 21 and $77 \%$ (33\%, median).

Quantification of IL-6 mRNA with qRT - PCR revealed a highly significant increase in the mean $I L-6 / A B L$ ratio in GBM $(33.3 \%)$ as compared with non-GBM (1.5\%; Kruskal-Wallis test, $\left.P=3.8 \times 10^{-6}\right)$. The mean $I L-6 / A B L$ ratio was $>10$-fold greater in GBM manifesting $I L-6$ gene amplification than in GBM without amplification (71.6 vs $6.1 \%$; $P=4.3 \times 10^{-7}$ ).

GBM patients with amplified $I L-6$ gene had significantly shorter survival than patients without amplification (log-rank test, $P=0.0000073$; Figure 1C). Multivariate Cox analysis for overall survival, including $I L-6$ amplification, extent of tumour resection and age as variables, demonstrated that $I L-6$ amplification was an independent factor of poor prognosis (relative risk (RR) amplified vs non-amplified $8.07 ; P=0.000016)$. The complete tumour resection, equally distributed among patients with and without $I L-6$ gene amplification, was an independent factor of favourable prognosis (RR complete $v s$ partial $0.31 ; P=0.013$ ). Age did not influence survival of patients.

\section{DISCUSSION}

Glioblastomas are the most devastating primary brain tumours. Despite modern treatments, about $40 \%$ of patients with GBM die within 6 months after diagnosis (Reardon et al, 2006). In the present study, we demonstrated that one of the molecular abnormalities associated with the aggressiveness of GBM is the amplification of the $I L-6$ gene, which was found in $41.7 \%$ of patients and significantly correlated with decreased survival.

Amplifications on chromosome $7 \mathrm{p}$ in GBM are believed to be driven by the amplification of the epidermal growth factor receptor (EGFR) gene locus (Rossi et al, 2005). This initial amplification event may induce instability along the length of this chromosomal arm, resulting in co-amplification of other genes. Of note, EGFR gene amplification has no clear prognostic value in GBM (Houillier et al, 2006). It may be possible that some of co-amplified (or independently amplified) genes are involved in glioma development and progression and are important for patient prognosis. Novel amplicons on chromosome $7 \mathrm{p}$ distinct from EGFR were recently identified using highresolution genomic analyses and were reported to contain, among other genes, the $I L-6$ gene that was overexpressed (Rossi et al, 2005).
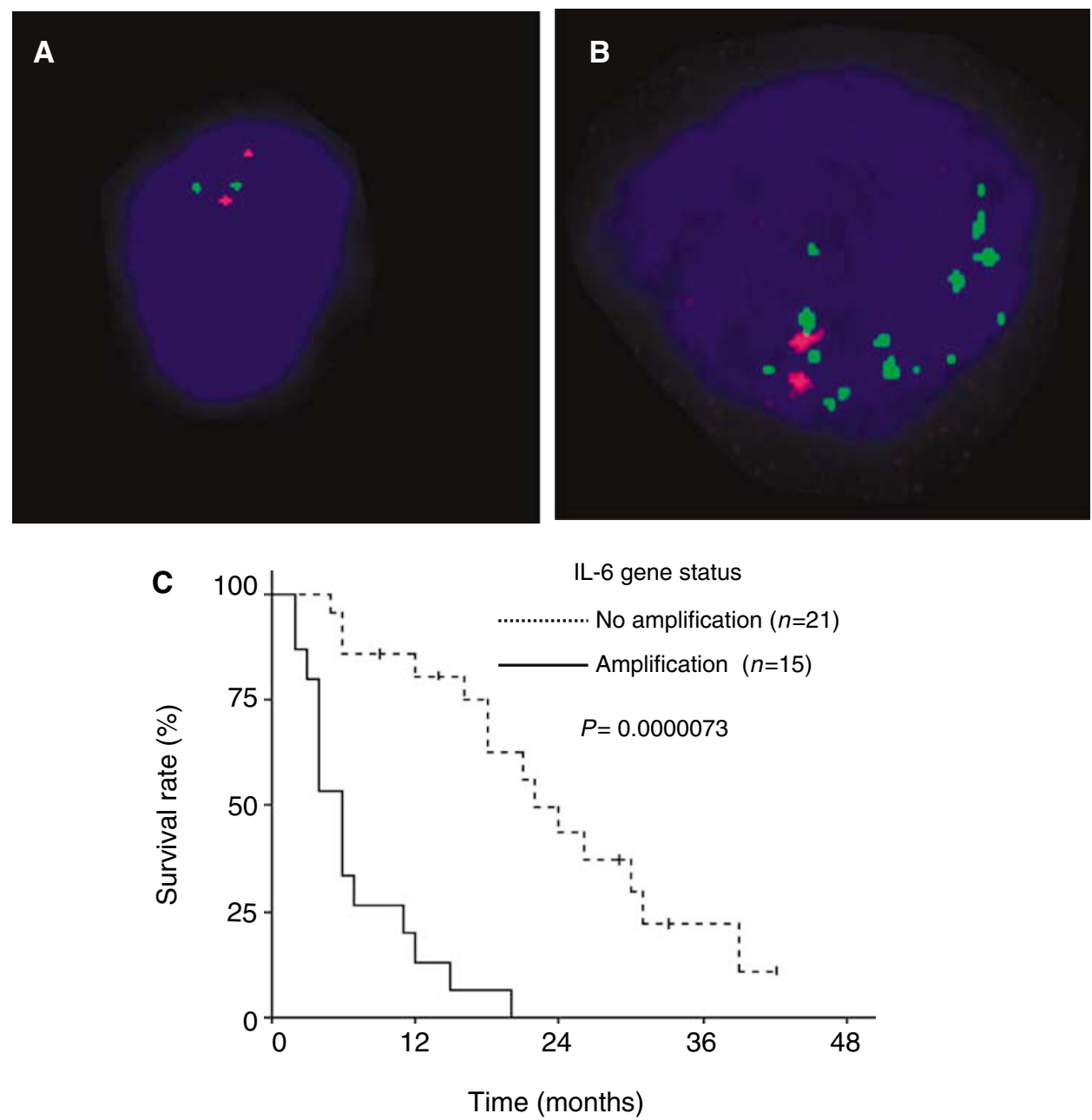

Figure I (A and B) Example of FISH analysis of chromosome 7 (red signals) and IL-6 gene (green signals) copy numbers in frozen sections of glioma tumours. A nucleus showing two copies of chromosome 7 and two copies of IL-6 gene (A); a nucleus with two copies of chromosome 7 and a high-level amplification of IL-6 gene (ratio of IL-6 to centromere 7 signals greater than 3) (B). (C) Overall survival as a function of IL-6 gene status in GBM patients. Patients with IL-6 gene amplification had significantly shorter survival than patients without amplification. 
In gliomas, IL-6 plays a role in promoting tumour growth (Goswami et al, 1998) and angiogenesis (Loeffler et al, 2005). This cytokine also protects cancer cells from apoptotic depletion during chemotherapy and radiotherapy through activation of the Janus kinase/STAT and phosphatidylinositol 3-kinase/AKT pathways (Miyamoto et al, 2001; Trikha et al, 2003; Hodge et al, 2005). In this context, amplification of the $I L-6$ gene leading to its overexpression is likely one of the major factors contributing to the aggressiveness and poor response to therapies of GBM. It may therefore be suggested that targeting IL-6 and its signalling

\section{REFERENCES}

Goswami S, Gupta A, Sharma SK (1998) Interleukin-6-mediated autocrine growth promotion in human glioblastoma multiforme cell line U87MG. J Neurochem 71: $1837-1845$

Hodge DR, Hurt EM, Farrar WL (2005) The role of IL-6 and STAT3 in inflammation and cancer. Eur J Cancer 41: 2502-2512

Houillier C, Lejeune J, Benouaich-Amiel A, Laigle-Donadey F, Criniere E, Mokhtari K, Thillet J, Delattre JY, Hoang-Xuan K, Sanson M (2006) Prognostic impact of molecular markers in a series of 220 primary glioblastomas. Cancer 106: $2218-2223$

Loeffler S, Fayard B, Weis J, Weissenberger J (2005) Interleukin-6 induces transcriptional activation of vascular endothelial growth factor (VEGF) in astrocytes in vivo and regulates VEGF promoter activity in glioblastoma cells via direct interaction between STAT3 and Sp1. Int J Cancer 115: $202-213$

Miyamoto Y, Hosotani R, Doi R, Wada M, Ida J, Tsuji S, Kawaguchi M, Nakajima S, Kobayashi H, Masui T, Imamura M (2001) Interleukin-6 inhibits radiation induced apoptosis in pancreatic cancer cells. Anticancer Res 21: $2449-2456$

Reardon DA, Rich JN, Friedman HS, Bigner DD (2006) Recent advances in the treatment of malignant astrocytoma. J Clin Oncol 24: 1253-1265

Rolhion C, Penault-Llorca F, Kemeny J-L, Lemaire J-J, Jullien C, LabitBouvier C, Finat-Duclos F, Verrelle P (2001) Interleukin-6 overexpression as a marker of malignancy in human glioma. J Neurosurg 94: 97-101

Rossi MR, La Duca J, Matsui S, Nowak NJ, Hawthorn L, Cowell JK. (2005) Novel amplicons on the short arm of chromosome 7 identified using pathways in GBM would sensitise these otherwise resistant tumours to chemotherapeutic drugs and radiotherapy.

\section{ACKNOWLEDGEMENTS}

This work was supported by the Ligue Nationale Contre le Cancer (Comité du Puy de Dôme). We are grateful to Georges Briançon and Farida Godeau for expert technical assistance and Fabrice Kwiatkowski for statistical analysis. high resolution array CGH contain over expressed genes in addition to EGFR in glioblastoma multiforme. Genes Chromosomes Cancer 44: $392-404$

Saigusa K, Hashimoto N, Tsuda H, Yokoi S, Maruno M, Yoshimine T, Aoyagi M, Ohno K, Imoto I, Inazawa J (2005) Overexpressed Skp2 within $5 \mathrm{p}$ amplification detected by array-based comparative genomic hybridization is associated with poor prognosis of glioblastomas. Cancer Sci 96: $676-683$

Suzuki T, Maruno M, Wada K, Kagawa N, Fujimoto Y, Hashimoto N, Izumoto S, Yoshimine T (2004) Genetic analysis of human glioblastomas using a genomic microarray system. Brain Tumor Pathol 21: 27-34

Tchirkov A, Rolhion C, Bertrand S, Doré JF, Dubost JJ, Verrelle P (2001) $I L-6$ gene amplification and expression in human glioblastoma. $\mathrm{Br} J$ Cancer 85: 518-522

Tchirkov A, Rolhion C, Kémény JL, Irthum B, Puget S, Khalil T, Chinot O, Kwiatkowski F, Périssel B, Vago P, Verrelle P (2003) Clinical implications of quantitative real-time RT-PCRanalysis of $h T E R T$ gene expression in human gliomas. $\mathrm{Br}$ J Cancer 88: 516-520

Trikha M, Corringham R, Klein B, Rossi JF (2003) Targeted antiinterleukin- 6 monoclonal antibody therapy for cancer: a review of the rationale and clinical evidence. Clin Cancer Res 9: 4653-4665

World Health Organization (2000) Astrocytic tumors. Oligodendroglial tumours and mixed gliomas. In: Pathology and Genetics of Tumours of the Nervous System Kleihues P, Cavenee WK (eds) pp 6-70. Lyon: IARCPress 